Investigação

\title{
Análise comparativa da performance mastigatória entre doentes portadores de prótese parcial removível acrílica e esquelética
}

\author{
Nuno Nóbrega*, Paulo Maurício \\ Instituto Superior de Ciências de Saúde Egas Moniz, Almada, Portugal
}

INFORMAÇÃO SOBRE O ARTIGO

Historial do artigo:

Recebido a 10 de Maio de 2017

Aceite a 29 de Setembro de 2017

On-line a 27 de Outubro de 2017

\section{Palauras-chave:}

Mastigação

Performance mastigatória

Prótese parcial removível

\section{R E S U M O}

Objetivos: Realizar uma análise comparativa da performance mastigatória entre os doentes portadores de prótese parcial removível acrílica e esquelética.

Métodos: A amostra foi constituída por 20 indivíduos, 10 portadores de prótese parcial removível acrílica bimaxilar (Grupo PA) e 10 portadores de prótese parcial removível esquelética bimaxilar (Grupo PE), com idades compreendidas entre os 49 e os 79 anos, sendo 12 do sexo feminino e 8 do sexo masculino. Neste estudo, foi utilizado um sistema de 7 peneiras granulométricas e foi confeccionado um alimento de origem artificial. Cada participante do estudo mastigou 20 cubos de alimento artificial, 10 cubos num $1 .^{\circ}$ ciclo mastigatório de 20 golpes e outros 10 cubos num 2. ${ }^{\circ}$ ciclo mastigatório de 40 golpes. Após a mastigação, cada paciente dispensou o alimento-teste num recipiente. Procedeu-se à análise granulométrica através do sistema de peneiras para determinar o diâmetro geométrico médio das partículas (DGM). Os resultados da amostra foram submetidos aos testes de normalidade; Kolmogorov-Smirnov e Shapiro-Wilk. Posteriormente foi efetuado o teste T-student para comparar as médias de DGM dos grupos PA e PE, com um nível de significância de 0,05. Resultados: Existem diferenças estatisticamente significativas entre as médias do DGM do grupo PA e do grupo PE, tanto para o $1 .^{\circ}$ ciclo mastigatório $(\mathrm{p}<0,001)$ como para o $2 .^{\circ}$ ciclo mastigatório $(\mathrm{p}<0,001)$. A média dos valores de $\mathrm{DGM}$ do grupo $\mathrm{PA}$ foi superior à do grupo $\mathrm{PE}$ nos dois ciclos mastigatórios.

Conclusão: Houve diferença da performance mastigatória entre os portadores de prótese parcial removível acrílica e esquelética. (Rev Port Estomatol Med Dent Cir Maxilofac. 2017;58(3):175-180)

(c) 2017 Sociedade Portuguesa de Estomatologia e Medicina Dentária. Publicado por SPEMD. Este é um artigo Open Access sob uma licença CC BY-NC-ND (http://creativecommons.org/licenses/by-nc-nd/4.0/).

\footnotetext{
${ }^{*}$ Autor correspondente.

Correio eletrónico: nunonobrega89@gmail.com (Nuno Filipe Nóbrega Silva).

http://doi.org/10.24873/j.rpemd.2017.10.025

1646-2890/@ 2017 Sociedade Portuguesa de Estomatologia e Medicina Dentária. Published by SPEMD.

This is an open access article under the CC BY-NC-ND license (http://creativecommons.org/licenses/by-nc-nd/4.0/).
} 


\title{
Comparative analysis of the masticatory performance between patients with acrylic versus metal removable partial dentures
}

\author{
A B S T R A C T
}

Keywords:

Chewing

Masticatory performance

Removable partial denture
Objectives: To perform a comparative analysis of the masticatory performance between patients with acrylic versus metal removable partial dentures.

Methods: 20 subjects participated in this study: 10 with a bimaxillary acrylic removable partial denture (PA group) and 10 with a bimaxillary metal removable partial denture (PE group). The subjects were aged between 49 and 79 years old and included 12 females and 8 males. A system of 7 granulometric sieves was employed, and an artificial food was elaborated. Each participant chewed 20 cubes of the artificial food: 10 cubes in a first masticatory cycle of 20 strokes and 10 cubes in a second masticatory cycle of 40 strokes. After chewing, each patient disposed of the food into a container. A particle size analysis, using a sieves system, was performed to determine the geometric mean particle diameter (GMD). The sample's results were submitted to the Kolmogorov-Smirnov and Shapiro-Wilk normality tests. The student t-test was then performed to compare the mean GMD in the PA and PE groups, considering a significance level of 0.05 .

Results: Statistically significant differences were verified between the mean GMD of the PA group and the PE group, both for the 1st masticatory cycle $(\mathrm{p}<0.001)$ and for the 2nd masticatory cycle $(p<0.001)$. The PA group's mean GMD values were higher than those of the PE group in the two masticatory cycles.

Conclusion: A difference was verified in the masticatory performance between patients with acrylic versus metal removable partial dentures. (Rev Port Estomatol Med Dent Cir Maxilofac. 2017;58(3):175-180)

(c) 2017 Sociedade Portuguesa de Estomatologia e Medicina Dentária. Published by SPEMD. This is an open access article under the CC BY-NC-ND license (http://creativecommons.org/licenses/by-nc-nd/4.0/).

\section{INTRODUÇÃO}

Com o crescimento da população geriátrica é expectável que haja um aumento da necessidade de próteses parciais removíveis. ${ }^{1,2}$ Atualmente, a utilização destas próteses para reabilitar os espaços edêntulos continua a ser uma opção de tratamento viável e previsível. ${ }^{3}$ Para além da estética e da fonética, estas próteses têm como propósito restabelecer a função mastigatória. ${ }^{4}$

Na literatura não existe consenso na termologia utilizada para descrever a função mastigatória. Os termos: performance, habilidade, eficiência e eficácia mastigatória são utilizados indiscriminadamente e em contextos diferentes. ${ }^{5,6}$ De acordo com o glossário dos termos de prostodontia, ${ }^{7}$ a performance mastigatória é a mensuração da fragmentação de um determinado alimento através de testes padronizados e a eficiência mastigatória é o esforço necessário para que um alimento alcance um determinado grau de fragmentação.

A medição da performance mastigatória pode ser realizada através da mastigação de um alimento que, posteriormente ao ciclo mastigatório é analisado em laboratório através de um sistema de peneiras granulométricas. $8,9,11,13-15$ Outros métodos para determinar o tamanho dos fragmentos encontram-se descritos na literatura, tais como: o método da colorimetria, ${ }^{12}$ o recurso à fotografia digital e aos programas de tratamento de imagem, ${ }^{10}$ scanners ópticos ${ }^{11,13}$ e gomas coloridas que mudam de cor com os golpes mastigatórios e que são calibradas por uma escala de cores ${ }^{16,20}$. Embora uma grande variedade de alimentos naturais e artificiais tenham sido descritos, $, 5,6,8-15$ na maioria dos estudos os autores optaram por confeccionar alimentos artificiais com recurso aos silicones de condensação para testar a performance mastigatória. 5,6,9,11,15

De acordo com o INM $^{17}$ (indicador normativo mastigatório), o limite do tamanho médio normal das partículas que compõem o bolo alimentar é $4000 \mu \mathrm{m}$. Valores superiores a este, são indicadores de uma reduzida função mastigatória. ${ }^{17} \mathrm{Num}$ outro estudo que avaliou a função mastigatória dos portadores de prótese parcial removível, concluíram que o diâmetro geométrico médio das partículas destes indivíduos permanece acima do indicador normativo mastigatório. ${ }^{18}$

O objetivo do presente estudo é realizar uma análise comparativa da performance mastigatória entre os doentes portadores de prótese parcial removível acrílica e esquelética, existindo a hipótese nula de não haver diferença da performance mastigatória entre os utilizadores de PPR acrílica e esquelética. 


\section{MATERIAIS E MÉTODOS}

O presente estudo foi realizado na clínica dentária Egas Moniz, situada no Monte de Caparica, Portugal, com os pacientes desta mesma clínica, após cada um ter aceite o consentimento informado. Foram seleccionados sequencialmente 20 indivíduos portadores de prótese parcial removível bimaxilar, com idades entre os 49 e os 79 anos, sendo 12 do sexo feminino e 8 do sexo masculino. Foram constituídos 2 grupos para este estudo. O primeiro grupo foi composto por 10 indivíduos portadores de prótese parcial removível acrílica bimaxilar (Grupo PA), 7 indivíduos do sexo feminino e 3 indivíduos do sexo masculino. O segundo grupo foi composto por 10 indivíduos portadores de prótese parcial removível esquelética bimaxilar (Grupo PE), 5 indivíduos do sexo masculino e 5 indivíduos do sexo feminino. Ambos os grupos enquadrados nos critérios de inclusão.

Neste estudo foram considerados os seguintes critérios de inclusão para os 2 grupos:

- Portadores de PPR com mais de 6 meses e menos de 5 anos;

- Portadores de PPR em que os dentes estão em oclusão (incluindo dentes naturais);

- Apresentar Classe I de Kennedy sem modificações na arcada superior e inferior;

- Presença de pelo menos 5 dentes posteriores (pelo menos 4 molares e 1 pré-molar) na PPR inferior e pelo menos 4 molares na PPR superior.

Neste estudo foram considerados os seguintes critérios de exclusão:

- Presença de disfunção temporomandibular;

- Presença de dor ou desconforto durante a mastigação;

- Indivíduos desconfortáveis com as suas PPR's.

Os testes de perfomance mastigatória foram realizados com um alimento de origem artificial composto por materiais dentários (Figura 1). O método e os materiais utilizados para a

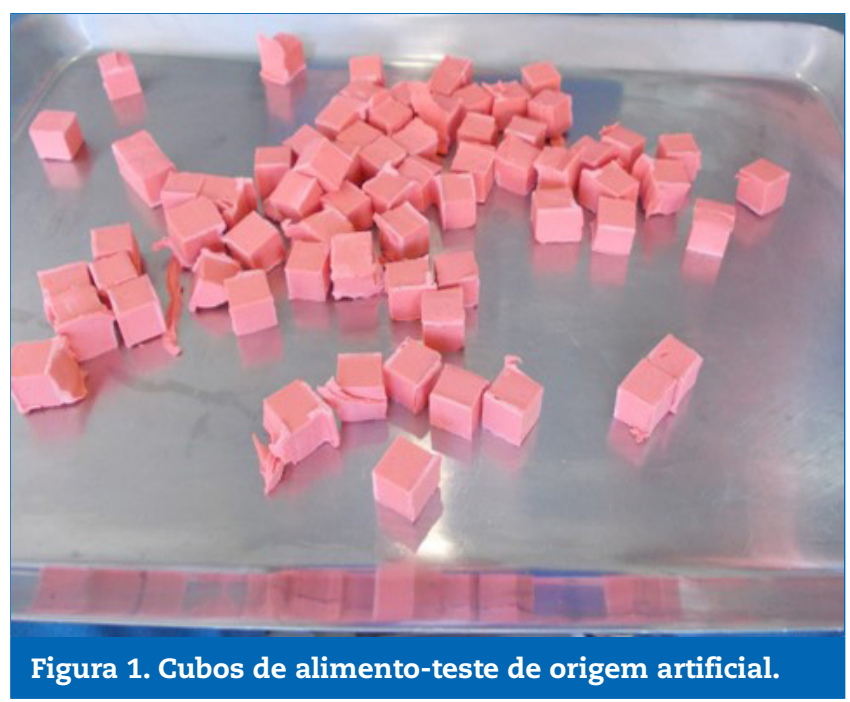

sua confecção foram adaptados de Slagter AP et al 1993. ${ }^{19}$ Para a sua confeção foram utilizados os seguintes compostos:

- 22,8g de silicone de adição (Normosil, Light ${ }^{\circledR}$ )

- $11 \mathrm{~g}$ de pasta de dentes Colgate Total ${ }^{\circledR}$

- $2 \mathrm{~g}$ de vaselina sólida,

$-2 \mathrm{~g}$ de alginato Ortoprint ${ }^{\circledR}$

$-4 \mathrm{~g}$ de gesso tipo IV

- 2 g de pasta catalisadora Perfil (Normosil, Putty ${ }^{\circledR}$ )

Todos os ingredientes foram anteriormente pesados com uma balança de precisão e foram misturados posteriormente até formarem uma massa homogénea. Foram colocados numa matriz pré-formada com 12,5 cm de lado e 0,68 cm de altura, contendo no seu interior formas cúbicas com $0,68 \mathrm{~cm}$ de lado. Para assegurar a completa polimerização, os moldes foram colocados numa estufa a $65^{\circ} \mathrm{C}$ durante 16 horas.

Antes de iniciar o teste de performance mastigatória, cada participante foi submetido a um exame clínico intra-oral, para a avaliação das mucosas e do grau de retenção e estabilidade das PPR's. Foi certificado que cada participante se enquadrava com os critérios de inclusão e exclusão. Foram fornecidos a cada participante deste estudo 20 cubos de alimento artificial. Solicitou-se a cada participante deste estudo que mastigasse 10 cubos em simultâneo e executasse um primeiro ciclo mastigatório com 20 golpes e posteriormente que mastigasse outros 10 cubos em simultâneo e executasse um segundo ciclo mastigatório com 40 golpes. Após cada ciclo mastigatório cada participante dispensou o alimento-teste para um recipiente e foi efetuada a análise desses fragmentos.

A análise dos fragmentos obtidos após a mastigação do alimento-teste foi realizada em laboratório através de um sistema de 7 peneiras granulométricas com diâmetros de abertura de $6,8 \mathrm{~mm}$; 4,0 mm; 2,8 $\mathrm{mm} ; 2,0 \mathrm{~mm} ; 1,4 \mathrm{~mm} ; 1,0 \mathrm{~mm}$ e 0,71 mm. Foi calculado o DGM (diâmetro geométrico médio) das partículas em $\mu \mathrm{m}$. O cálculo do DGM de cada amostra foi obtido através do software de granulometria Granucalc 1.0. Para cada amostra, foi inserido no software o peso dos fragmentos retidos e o diâmetro da peneira correspondente, sendo dado automaticamente, o valor do DGM em $\mu \mathrm{m}$ e o respectivo desvio padrão geométrico DPG (Tabela 1 e 2). Quanto maiores forem os valores de DGM, menor será a eficácia mastigatória e vice-versa. Após a análise granulométrica procedeu-se ao tratamento estatístico dos dados obtidos utilizando o software SPSS ${ }^{\circledR}$ versão 22.0 para Windows ${ }^{\circledR}$.

Para averiguar se a amostra tem uma distribuição normal, os resultados foram submetidos aos testes de normalidade: Kolmogorov-Smirnov e Shapiro-Wilk. Tendo sido obtido $p>0,2$ nos testes de normalidade, este valor permite-nos aceitar a hipótese de normalidade da população, sendo esta aceite para valores $p>0,05$. Posteriormente, foi efetuado o teste T-student com 2 amostras independentes para comparar as médias dos DGM das partículas dos grupos PA e PE. Para a comparação dos DGM das partículas dentro de cada grupo após o $1 .^{\circ}$ e o $2 .^{\circ}$ ciclos mastigatórios, procedeu-se ao teste T-student com 2 amostras emparelhadas. Em ambas as análises comparativas, foi utilizado um nível de significância de 0,05.

Para o cálculo da redução (R) que as partículas sofreram durante a mastigação, foi necessário calcular o DGM máximo. 
Tabela 1. Resultados obtidos após o peneiramento das amostras do grupo PA; 6,8 mm; 4,0 mm;2,8 $\mathrm{mm} ; 2,0 \mathrm{~mm} ; 1,4 \mathrm{~mm}$; $1 \mathrm{~mm} ; 0,71 \mathrm{~mm}$, estes valores correspondem ao diâmetro de abertura das peneiras utilizadas. DGM corresponde ao diâmetro geométrico médio das partículas e DPG o desvio padrão geométrico.

\begin{tabular}{|c|c|c|c|c|c|c|c|c|c|}
\hline Grupo PA & $6,8 \mathrm{~mm}$ & $4,0 \mathrm{~mm}$ & $2,8 \mathrm{~mm}$ & $2,0 \mathrm{~mm}$ & $1,4 \mathrm{~mm}$ & $1,0 \mathrm{~mm}$ & $0,71 \mathrm{~mm}$ & DPG & DGM \\
\hline 1-A & 19.7086 & 16.5082 & 0.0000 & 0.0000 & 0.0000 & 0.0000 & 0.0000 & 1.18 & 7420 \\
\hline 1-B & 19.1496 & 16.5082 & 14.8984 & 0.0000 & 0.0000 & 0.0000 & 0.0000 & 1.23 & 7016 \\
\hline 2-A & 19.7686 & 15.8574 & 0.0000 & 0.0000 & 0.0000 & 0.0000 & 0.0000 & 1,19 & 7379 \\
\hline 2-B & 19.1955 & 15.7720 & 14.8420 & 0.0000 & 0.0000 & 0.0000 & 0.0000 & 1.19 & 7362 \\
\hline 3-A & 19.1940 & 16.7302 & 0.0000 & 0.0000 & 0.0000 & 0.0000 & 0.0000 & 1.22 & 7034 \\
\hline 3-B & 18.0872 & 17.2859 & 15.2454 & 0.0000 & 0.0000 & 0.0000 & 0.0000 & 1.30 & 6407 \\
\hline 4-A & 19.3271 & 16.5760 & 0.0000 & 0.0000 & 0.0000 & 0.0000 & 0.0000 & 1.09 & 7847 \\
\hline 4-B & 20.4162 & 15.0774 & 0.0000 & 0.0000 & 0.0000 & 0.0000 & 0.0000 & 1.10 & 7813 \\
\hline 5-A & 19.3423 & 16.6490 & 0.0000 & 0.0000 & 0.0000 & 0.0000 & 0.0000 & 1.18 & 7527 \\
\hline 5-B & 19.4102 & 15.8678 & 0.0000 & 0.0000 & 0.0000 & 0.0000 & 0.0000 & 1.18 & 7421 \\
\hline 6-A & 21.1170 & 0.0000 & 0.0000 & 0.0000 & 0.0000 & 0.0000 & 0.0000 & 1.00 & 7998 \\
\hline 6-B & 20.5483 & 0.0000 & 0.0000 & 0.0000 & 0.0000 & 0.0000 & 0.0000 & 1.00 & 7998 \\
\hline 7-A & 20.7643 & 0.0000 & 0.0000 & 0.0000 & 0.0000 & 0.0000 & 0.0000 & 1.00 & 7998 \\
\hline 7-B & 20.6956 & 0.0000 & 0.0000 & 0.0000 & 0.0000 & 0.0000 & 0.0000 & 1.00 & 7998 \\
\hline 8-A & 19.6542 & 15.4454 & 0.0000 & 0.0000 & 0.0000 & 0.0000 & 0.0000 & 1.14 & 7665 \\
\hline 8-B & 20.5163 & 15.7351 & 0.0000 & 0.0000 & 0.0000 & 0.0000 & 0.0000 & 1.14 & 7574 \\
\hline 9-A & 19.7955 & 15.3345 & 14.9463 & 0.0000 & 0.0000 & 0.0000 & 0.0000 & 1.13 & 7717 \\
\hline 9-B & 19.5730 & 16.7561 & 15.3013 & 0.0000 & 0.0000 & 0.0000 & 0.0000 & 1.30 & 6645 \\
\hline 10-A & 21.2606 & 15.1889 & 15.1000 & 0.0000 & 0.0000 & 0.0000 & 0.0000 & 1.05 & 7949 \\
\hline 10-B & 20.5608 & 15.3000 & 15.1000 & 0.0000 & 0.0000 & 0.0000 & 0.0000 & 1.06 & 7943 \\
\hline
\end{tabular}

Tabela 2. Resultados obtidos após o peneiramento das amostras do grupo PE; 6,8 mm; 4,0 mm;2,8 $\mathrm{mm} ; 2,0 \mathrm{~mm} ; 1,4 \mathrm{~mm}$; $1 \mathrm{~mm} ; 0,71 \mathrm{~mm}$, estes valores correspondem ao diâmetro de abertura das peneiras utilizadas. DGM corresponde ao diâmetro geométrico médio das partículas e DPG o desvio padrão geométrico.

\begin{tabular}{|c|c|c|c|c|c|c|c|c|c|}
\hline Grupo PE & $6,8 \mathrm{~mm}$ & $4,0 \mathrm{~mm}$ & $2,8 \mathrm{~mm}$ & $2,0 \mathrm{~mm}$ & $1,4 \mathrm{~mm}$ & $1,0 \mathrm{~mm}$ & $0,71 \mathrm{~mm}$ & DPG & DGM \\
\hline $1-A$ & 20.3400 & 15.6180 & 14.9989 & 15.1000 & 14.9989 & 14.9989 & 14.9948 & 1.74 & 6168 \\
\hline 1-B & 19.6326 & 16.3261 & 15.5728 & 15.5122 & 14.8840 & 14.8253 & 14.8200 & 1.71 & 5480 \\
\hline 2-A & 18.6596 & 16.9683 & 16.6274 & 15.0221 & 0.0000 & 0.0000 & 0.0000 & 1.41 & 5997 \\
\hline 2-B & 18.0039 & 16.5973 & 16.3247 & 15.0383 & 15.0072 & 14.9999 & 14.9969 & 1.44 & 5800 \\
\hline 3-A & 18.6495 & 16.3639 & 0.0000 & 0.0000 & 0.0000 & 0.0000 & 0.0000 & 1.21 & 7137 \\
\hline 3-B & 18.7844 & 16.3976 & 0.0000 & 0.0000 & 0.0000 & 0.0000 & 0.0000 & 1.22 & 7130 \\
\hline 4-A & 17.7131 & 16.5958 & 14.9754 & 14.6406 & 0.0000 & 0.0000 & 0.0000 & 1.30 & 6467 \\
\hline 4-B & 18.7544 & 16.4374 & 14.8595 & 14.7610 & 14.7272 & 14.6595 & 14.6562 & 1.45 & 6346 \\
\hline 5-A & 19.1371 & 16.4384 & 0.0000 & 0.0000 & 0.0000 & 0.0000 & 0.0000 & 1.21 & 7087 \\
\hline 5-B & 16.9629 & 17.0254 & 0.0000 & 0.0000 & 0.0000 & 0.0000 & 0.0000 & 1.00 & 6423 \\
\hline $6-A$ & 18.5515 & 17.1988 & 0.0000 & 0.0000 & 0.0000 & 0.0000 & 0.0000 & 1,23 & 6769 \\
\hline 6-B & 17.3168 & 18.8623 & 15.0511 & 0.0000 & 0.0000 & 0.0000 & 0.0000 & 1.25 & 6023 \\
\hline 7-A & 19.0161 & 16.3924 & 15.1821 & 0.0000 & 0.0000 & 0.0000 & 0.0000 & 1.30 & 6777 \\
\hline 7-B & 16.4423 & 18.8620 & 15.2187 & 14.9106 & 14.8134 & 14.8108 & 14.8106 & 1.36 & 5463 \\
\hline 8-A & 20.7409 & 16.4038 & 16.2644 & 0.0000 & 0.0000 & 0.0000 & 0.0000 & 1.39 & 6507 \\
\hline 8-B & 18.5795 & 17.2417 & 15.5654 & 15.2234 & 15.0381 & 15.0070 & 14.9979 & 1.38 & 6065 \\
\hline 9-A & 15.7487 & 20.2756 & 15.2954 & 0.0000 & 0.0000 & 0.0000 & 0.0000 & 1.20 & 5346 \\
\hline 9-B & 15,5242 & 20.2497 & 0.0000 & 15.2172 & 15.0223 & 0.0000 & 0.0000 & 1.35 & 4062 \\
\hline $10-A$ & 18.0120 & 17.9703 & 15.0234 & 0.0000 & 0.0000 & 0.0000 & 0.0000 & 1.26 & 6382 \\
\hline 10-B & 15.8042 & 19.8639 & 15.8072 & 15.2047 & 0.0000 & 0.0000 & 0.0000 & 1.31 & 5025 \\
\hline
\end{tabular}


Para a obtenção do DGM máximo, foi utilizado o software Granucalc 1.0, simulando uma situação em que todo o alimento ficaria retido na peneira granulométrica de maior diâmetro de abertura, sendo a peneira de $6,8 \mathrm{~mm}$ a peneira de maior diâmetro. O DGM máximo obtido foi de 7998 um. Para o cálculo da redução $(\mathrm{R})$ que as partículas sofreram após os ciclos mastigatórios, utilizou-se a seguinte equação:

Equação 1: R = 7998 - DGM (do grupo de estudo)

\section{RESULTADOS}

Os resultados obtidos demonstram que existem diferenças estatisticamente significativas entre a média dos valores de DGM do grupo PA e do grupo PE tanto no $1 .^{\circ}$ ciclo mastigatório ( $p<0,001$, teste t-Student) como no $2 .^{\circ}$ ciclo mastigatório $(p<$ 0,001 , teste t-Student). A média dos valores de DGM do grupo PA foi superior à do grupo PE em ambos os ciclos mastigatórios (Tabela 3).

Tabela 3. Média dos DGM's e o respetivo desvio padrão obtido pelo grupo PA e pelo grupo PE após o $10^{\circ}$ e $020^{\circ}$ ciclo mastigatório.

\begin{tabular}{ccc} 
Grupo & $\mathbf{1 .}^{\circ}$ ciclo & 2. ${ }^{\circ}$ ciclo \\
\hline PA & $7653 \mu \mathrm{m} \pm 315 \mu \mathrm{m}$ & $7418 \mu \mathrm{m} \pm 569 \mu \mathrm{m}$ \\
\hline PE & $6463 \mu \mathrm{m} \pm 537 \mu \mathrm{m}$ & $5782 \mu \mathrm{m} \pm 844 \mu \mathrm{m}$ \\
\hline
\end{tabular}

No grupo PA, não existiu uma diferença estatisticamente significativa entre a média dos valores de DGM do $1 .^{\circ}$ e a do $2 .^{\circ}$ ciclo mastigatório ( $p=0,069$, teste t-Student, amostras emparelhadas). Contrariamente, no grupo PE existiu uma diferença estatisticamente significativa entre a média dos valores de DGM do $1 .^{\circ}$ e a do $2 .^{\circ}$ ciclo mastigatório ( $p=0,002$, teste t-Student, amostras emparelhadas).

O valor médio da redução das partículas do grupo PA após o $1 .^{\circ}$ ciclo mastigatório foi de $345 \mu \mathrm{m}$ e após o $2 .^{\circ}$ ciclo mastigatório foi de $580 \mu \mathrm{m}$. Correspondendo a uma eficácia mastigatória de 4,3\% e 7,3\% respetivamente. O valor médio da redução das partículas do grupo PE após o $1 .^{\circ}$ ciclo mastigatório foi de $1535 \mu \mathrm{m}$ e após o 2..$^{\circ}$ ciclo mastigatório foi de $2216 \mu \mathrm{m}$. Correspondendo a uma eficácia mastigatória de 19,1\% e 27,7\% respetivamente.

\section{DISCUSSÃO}

Neste estudo, para a realização dos testes de performance mastigatória optou-se por confeccionar um alimento artificial. Neste tipo de testes, os alimentos artificiais apresentam vantagens em relação aos naturais por serem reprodutíveis e padronizáveis. ${ }^{19}$ Embora vários métodos tenham sido descritos na literatura, ${ }^{5,6,8-16,20}$ novos estudos ${ }^{16,20}$ sugerem que a utilização de gomas que mudam de cor com os golpes mastigatórios e que são calibradas através de uma escala de cores, poderá ser o método mais indicado para medir a performance mastigatória. Porém esse método ainda carece de estudos clíni- cos. ${ }^{16,20}$ Neste estudo, optou-se pela utilização de um sistema de peneiras granulométricas para determinar o tamanho das partículas. Este é sem dúvida o método mais utilizado pelos autores sendo considerado o método standard para determinar a performance mastigatória. ${ }^{8} \mathrm{~A}$ utilização de múltiplas peneiras granulométricas permite uma medição mais precisa quando comparado com o método de uma única peneira. ${ }^{9} \mathrm{O}$ método de uma única peneira só fornece resultados confiáveis da performance mastigatória quando o DGM das partículas é próximo do diâmetro de abertura da peneira. ${ }^{9}$

De acordo com outros estudos, ${ }^{21,22}$ o fluxo salivar, a força de mordida e a área oclusal são fatores que influenciam a performance mastigatória. Se a perda dentária e a hipossialia não é uma consequência fisiológica da idade, então a idade não é considerada um fator determinante para a performance mastigatória. ${ }^{22}$

Na nossa amostra, o grupo PA apresenta um maior número de indivíduos do género feminino que o grupo PE, investigações anteriores ${ }^{21,22}$ indicam que a diferença dos géneros não influência os resultados da performance mastigatória. Parece que embora os indivíduos do género feminino tenham uma menor força muscular comparativamente aos do género masculino, essa força muscular é compensada por uma melhor coordenação e atividade sensorial por parte dos indivíduos do género feminino. ${ }^{22}$

Neste estudo, um dos critérios de inclusão para os portadores de prótese parcial removível bimaxilar, foi o tempo de uso da prótese, embora o intervalo definido varie entre os 6 meses e os 5 anos, esse intervalo de acordo com estudos anteriores ${ }^{23,24}$ parece não influenciar os resultados. A maioria das PPR's continuam a apresentar uma boa retenção e estabilidade após 5 anos de utilização. ${ }^{23,24}$ Por outro lado, um exame clínico prévio foi realizado neste estudo de forma avaliar a estabilidade e retenção das PPR's dos participantes. As PPR's que apresentavam algum tipo de deslocamento vertical e/ou horizontal foram excluídas deste estudo. Porém detectamos 2 limitações neste estudo: a fadiga muscular que os participantes poderiam apresentar no $2 .^{\circ}$ ciclo mastigatório e a pequena variação do número de pré-molares entre os portadores de PPR's bimaxilares.

Neste estudo verificou-se que os resultados obtidos para a prótese acrílica foram semelhantes aos resultados obtidos para as próteses totais muco-suportadas de um outro estudo, ${ }^{25}$ com uma performance mastigatória de apenas $5 \%$. Os valores semelhantes da performance mastigatória encontrados entre os portadores de PPR's acrílicas bimaxilares com extremidades livres e os portadores de próteses totais muco-suportadas, poderão estar relacionados com o seu mecanismo de suporte e a ausência de dentes remanescentes posteriores. Os resultados obtidos para a PPR esquelética no presente estudo estão em concordância com os resultados de um outro estudo ${ }^{18}$ em que os portadores de PPR esquelética também apresentaram valores de DGM acima dos $4000 \mu \mathrm{m}$. Verificou-se também que neste estudo todos os participantes do grupo PA (Tabela 1) e do grupo PE (Tabela 2) obtiveram um valor de DGM superior ao $\operatorname{INM}^{17}(4000 \mu \mathrm{m})$ tanto no $1 .^{\circ}$ ciclo como no $2 .^{\circ}$ ciclo mastigatório. Estes valores permitem afirmar que todos os participantes deste estudo apresentaram perturbações na função mastigatória. 
Os portadores de PPR acrílica bimaxilar apresentaram os piores resultados de performance mastigatória deste estudo. Parece que os portadores de PPR acrílica mesmo com o aumento do número de golpes durante a mastigação, não conseguem compensar a sua diminuída performance mastigatória, havendo uma tendência por parte destes usuários a deglutir fragmentos maiores de alimento. Já os portadores de PPR esquelética, tendem a melhorar a sua performance mastigatória com o aumento do número de golpes durante a mastigação.

\section{CONCLUSÃO}

Verificou-se que houve diferença da performance mastigatória entre os portadores de prótese parcial removível acrílica e esquelética. Os portadores de prótese parcial removível esquelética possuem uma melhor performance mastigatória comparativamente aos portadores de prótese parcial removível acrílica.

\section{Responsabilidades éticas}

Proteção de pessoas e animais. Os autores declaram que os procedimentos seguidos estavam de acordo com os regulamentos da comissão de investigação clínica e ética relevante e de acordo com os do Código de Ética da Associação Médica Mundial (Declaração de Helsínquia).

Confidencialidade dos dados. Os autores declaram ter seguido os protocolos do seu centro de trabalho acerca da publicação dos dados de pacientes.

Direito à privacidade e consentimento escrito. Os autores declaram ter recebido consentimento escrito dos pacientes e/ ou sujeitos mencionados no artigo. O autor para correspondência está na posse deste documento.

\section{Conflito de interesses}

Os autores declaram não haver conflito de interesses.

\section{B I B L I O G R A F I A}

1. Jones JD, Turkyilmaz I, Garcia LT. Removable partial dentures - treatment now and for the future. Tex Dent J. 2010;127:365-72.

2. Taheri JB, Mortazavi H, Vahid-Dastjerdi E, Maleki Z, Parsaee P. The frequency of dental prostheses in young, adult and older individuals living in Tehran, Iran. Int J Dent Clinics. 2012;4:1-5.

3. Xie Q Ding T, Yang G. Rehabilitation of oral function with removable dentures still an option? J Oral Rehabil. 2015;42:234-42.

4. Roumanas ED. The Social Solution-Denture Esthetics, Phonetics, and Function. J Prosthodont. 2009;18:112-5.

5. Eberhard L, Schindler HJ, Hellmann D, Schmitter M, Rammelsberg P, Giannakopoulos NN. Comparison of particle-size distributions determined by optical scanning and by sieving in the assessment of masticatory performance. J Oral Rehabil. 2012;39:338-48.

6. Mendonça DB, Mendes FA, Mendonça G. Comparison of Masticatory Function Between Subjects with Three Types of Dentition. Int J Prosthodont. 2009;22:399-404.

7. The glossary of prosthodontic terms, Ninth Edition. J Prosthet Dent. 2005;94:10-92.

8. Katsuhiko k, Takahiro O, Garrett NR, Minoru T. Assesment of masticatory performance - Methodologies and their application. Prosthodont Res Pract. 2004;3:33-45.

9. Van der Bilt A, Fontijn-Tekamp FA. Comparison of single and multiple sieve methods for the determination of masticatory performance. Archives of Oral Biology. 2004;49:193-8.

10. Sugimoto K, Iegami CM, Iida S, Naito M, Tamaki R, Minagi S. New image analysis of large food particles can discriminate experimentally suppressed mastication. J Oral Rehabil. 2012;39:405-10.

11. Eberhard L, Schneider S, Eiffler C, Kappel S, Giannakopoulos NN. Particle size distributions determined by optical scanning and by sieving in the assessment of masticatory performance of complete denture wearers. Clin Oral Invest. 2015;19:429-36.

12. Kayser AF, van der Hoeven JS. Colorimetric determinationof the masticatory performance. J Oral Rehabil. 1977;4:145-8.

13. Van der Bilt A, Mojet J, Tekamp FA, Abbink JH. Comparing masticatory performance and mixing ability. J Oral Rehabil. 2010;37:79-84.

14. AL-ALI F, Heath MR, Wright PS. Simplified method of estimating masticatory performance. J Oral Rehabil. 1999;26:678-83.

15. Oliveira RL, Noronha WP, Bonjardim LR. Avaliação Da Performance Mastigatória em indivíduos respiradores orais e nasais. rev CEFAC. 2012;14:114-21.

16. Tarkowska A, Katzer L, Ahlers MO. Assessment of masticatory performance by means of a color-changeable chewing gum. JProsthodont Res. 2017;61:9-19.

17. Woda A, Nicolas E, Mishellany A, Hennequin M, Mazille MN, Veyrune JL. Peyron MA. The Masticatory Normative Indicator. J Dent Res. 2010;89:281-5.

18. Bessadet M, Nicolas E, Sochat M, Hennequin M, Veyrune JL. Impact of removable partial denture prosthesis on chewing efficiency. J Appl Oral Sci. 2013;21:392-6.

19. Slagter AP, Bosman F, Van der Bilt A. Comminution of two artificial test foods by dentate and edentulous subjects. J Oral Rehabil. 1993;20:159-76.

20. Ishikawa Y, Watanabe I, Hayakawa I, Minakuchi S, Uchida T. Evaluations of Masticatory Performance of Complete Denture Wearers Using. Color-Changeable Chewing Gum and Other Evaluating Methods. J Med Dent Sci. 2007;54:65-70.

21. Hatch JP, Shinkai RS, Sakai S, Rugh JD, Paunovich ED. Determinants of masticatory performance in dentate adults. Arch Oral Biol. 2001;46:641-8.

22. Ikebe K, Matsuda K, Kagawa R, Enoki K, Yoshida M, Maeda Y, Nokubi T. Association of masticatory performance with age, gender, number of teeth, occlusal force and salivary flow in Japanese older adults: is ageing a risk factor for masticatory dysfunction? Arch Oral Biol. 2011;56:991-6.

23. Yeung AL, Lo EC, Clark RK, Chow TW. Usage and status of cobalt-chromium removable partial dentures 5-6 years after placement. J Oral Rehabil. 2002;29:127-32.

24. Cosme DC, Baldisserotto SM, Fernandes Ede L, Rivaldo EG, Rosing CK, Shinkai RS. Functional evaluation of oral rehabilitation with removable partial dentures after five years. J Appl Oral Sci. 2006;14:111-6.

25. Ow RK, Carlsson GE, Karlsson S. Relationship of masticatory mandibular movements to masticatory performance of dentate adults: a method study. J Oral Rehabil. 1998;25:821-9. 